\title{
Special section on spatial and temporal databases
}

\author{
Nikos Mamoulis • Thomas Seidl
}

Published online: 6 September 2011

(c) Springer Science+Business Media, LLC 2011

This section of the issue hosts extended versions of the four best papers of the 11th International Symposium on Spatial and Temporal Databases (SSTD 2009). The proceedings of the symposium are published by Springer, as volume 5644 of the series Lecture Notes in Computer Science. More information about it can be found at http://www.springeronline.com/978-3-642-02981-3.

For the first time in the SSTD series, the best paper of the symposium was awarded and a few high quality papers were selected and the authors were invited to submit extended versions of their work to Geoinformatica.

The paper Spatial Skyline Queries: Exact and Approximation Algorithms, by MuWoong Lee, Wanbin Son, Hee-Kap Ahn, Seung-won Hwang, is an extension of the paper "Spatial Skyline Queries: An Efficient Geometric Algorithm," by the same authors, which received the best paper award at the symposium. The paper deals with spatial skyline; a query which retrieves the subset of objects in space that are not 'spatially dominated' by others with respect to their distances to a set of pivot points. The authors propose a simple and efficient algorithm for spatial skyline evaluation, which correctly computes the result and at the same time it is very efficient compared to previous solutions. Compared to the paper presented at the symposium, the extended version includes additional analytical results and comparison experiments with previous work. In addition, an extension of the proposed approximation algorithm is developed to trace skyline changes, for the case where one of the query points moves in one direction.

\footnotetext{
N. Mamoulis $(\varangle)$

Department of Computer Science, University of Hong Kong, Pokfulam Road, Hong Kong, Hong Kong e-mail:nikos@cs.hku.hk

T. Seidl

Department of Computer Science 9, RWTH Aachen University, 52056 Aachen, Germany
} 
The paper Editing and Versioning for High Performance Network Models in a Multiuser Environment, by Petko Bakalov, Erik Hoel, Wee-Liang Heng, Sudhakar Menon, and Vassilis J. Tsotras, is an extension of the SSTD09 paper "Versioning of Network Models in a Multiuser Environment." The paper provides a unified solution to the problems of dynamic editing and versioning of network models. Network data models are used as a mechanism for describing connectivity between spatial features in GIS applications. The authors propose an efficient algorithm that incrementally maintains connectivity within a dynamic network. Compared to the paper presented at the symposium, the extended version includes a 'hyperedge' extension of the basic network model, which drastically decreases the number of edge elements accessed during solve time on large networks; this in turn leads to faster solve operations. In addition, the authors propose a new network model versioning scheme that uses flexible reconciling rules.

The paper MOVIES: Indexing Moving Objects by Shooting Index Images, by Jens Dittrich, Lukas Blunschi, and Marcos Vaz Salles, extends "Indexing Moving Objects using Short-Lived Throwaway Indexes." The authors follow an unconventional approach to indexing moving objects; instead of using a single index that is updated at every timestamp with the changes in object positions, they construct conceptually simple shortlived index images that we only keep for a very short period of time (subseconds) in main memory. In this way, at the same time high query rates and high update rates are supported. Compared to the paper presented at the symposium, the extended version includes a quality-aware optimization for the index, as well as a wide range of additional experiments that evaluate its performance under different conditions.

The paper Approximate and Exact Hybrid Algorithms for Private NearestNeighbor Queries with Database Protection, by Gabriel Ghinita, Panos Kalnis, Murat Kantarcioglu, and Elisa Bertino, extends SSTD09 paper "A Hybrid Technique for Private Location-Based Queries with Database Protection." The authors deal with protecting the location privacy of mobile users, when the latter issue queries that retrieve nearby points of interest. The paper proposes hybrid, two-step approaches for private location-based queries, which rely on private information retrieval protocols and cryptographic techniques. Compared to the SSTD paper, this extended version includes a new protocol for private evaluation of point-in-convex-polygon enclosure, which can be used to greatly improve the performance of private hybrid exact NN queries. In addition, the experimental section is reorganized and includes additional experiments.

The best papers from SSTD 2009 were carefully selected after reading the reviewer comments and recommendation. In the fall of 2009, we informed the authors and invited them to submit extended versions of their works to Geoinformatica. We invited four reviewers per paper, including whenever possible the ones that reviewed the corresponding SSTD 2009 submissions of the papers. After few rounds of reviews, we accepted all four invited submissions for publication. In response to the review comments, the authors of the published papers significantly improved their work. We would like to thank both the authors and their reviewers for their outstanding efforts.

Nikos Mamoulis

Thomas Seidl

PC co-chairs of SSTD 2009

Guest editors of Geoinformatica 